\title{
Inhibition of tumour necrosis factor- $\alpha$ and interleukin 6 production by mononuclear cells following dietary fish-oil supplementation in healthy men and response to antioxidant co-supplementation
}

\author{
Timothy Trebble ${ }^{1 \star}$, Nigel K. Arden ${ }^{2}$, Mike A. Stroud ${ }^{1}$, Stephen A. Wootton ${ }^{1}$, Graham C. Burdge ${ }^{1}$, \\ Elizabeth A. Miles ${ }^{1}$, Anne B. Ballinger ${ }^{3}$, Rachel L. Thompson ${ }^{1}$ and Philip C. Calder ${ }^{1}$ \\ ${ }^{1}$ The Institute of Human Nutrition, School of Medicine, University of Southampton, Tremona Road, \\ Southampton SO16 6YD, UK \\ ${ }^{2}$ MRC Environmental Epidemiology Unit, University of Southampton, Tremona Road, \\ Southampton General Hospital, Southampton SO16 6YD, UK \\ ${ }^{3}$ Department of Adult and Paediatric Gastroenterology, Barts and the London, \\ Queen Mary's School of Medicine and Dentistry, University of London, London E1 2AD, UK
}

(Received 4 July 2002 - Revised 27 February 2003 - Accepted 13 March 2003)

\begin{abstract}
Increased dietary consumption of the $n-3$ polyunsaturated fatty acids (PUFA) eicosapentaenoic acid (20:5n-3; EPA) and docosahexaenoic acid (22: 6n-6; DHA) is associated with their incorporation into circulating phospholipid and increased production of lipid peroxide metabolites. The relationship between peripheral blood mononuclear cell (PBMC) function, $n$-3 PUFA intake and antioxidant co-supplementation is poorly defined. We therefore investigated tumour necrosis factor (TNF)- $\alpha$ and interleukin (IL) 6 production by PBMC and phospholipid fatty acid composition in plasma and erythrocytes of healthy male subjects $(n$ 16) receiving supplemental intakes of $0 \cdot 3,1.0$ and $2.0 \mathrm{~g}$ EPA + DHA/d, as consecutive 4-week courses. All subjects were randomised in a double-blind manner to receive a concurrent antioxidant supplement ( $200 \mu \mathrm{g} \mathrm{Se}, 3 \mathrm{mg} \mathrm{Mn}, 30 \mathrm{mg}$ D- $\alpha$-tocopheryl succinate, $90 \mathrm{mg}$ ascorbic acid, $450 \mu \mathrm{g}$ vitamin A ( $\beta$-carotene and retinol)) or placebo. There was a positive dose-dependent relationship between dietary $n-3$ PUFA intake and EPA and DHA incorporation into plasma phosphatidylcholine and erythrocyte phosphatidylethanolamine, with a tendency towards a plateau at higher levels of intake. Production of TNF- $\alpha$ and IL- 6 by PBMC decreased with increasing $n-3$ PUFA intake but tended towards a 'Ushaped' dose response. Both responses appeared to be augmented by antioxidant co-supplementation at intermediate supplementary $n$ - 3 PUFA intakes. Thus, increased dietary $n-3$ PUFA consumption resulted in defined but contrasting patterns of modulation of phospholipid fatty acid composition and PBMC function, which were further influenced by antioxidant intake.
\end{abstract}

Fish oil: Antioxidants: Tumour necrosis factor- $\alpha$ : Interleukin 6

The cytokines tumour necrosis factor (TNF)- $\alpha$ and interleukin (IL) 6 are soluble, locally released inflammatory mediators credited with a multitude of regulatory roles. TNF- $\alpha$ has a pivotal position within the immune response as demonstrated by the marked, clinically evident, efficacy of anti-TNF- $\alpha$ monoclonal antibody therapies in rheumatoid arthritis and Crohn's disease (Elliott et al. 1995; Targan et al. 1997). Human peripheral blood mononuclear cells (PBMC), which typically comprise $10-15 \%$ monocytes and $85-90 \%$ $\mathrm{T}$ and B lymphocytes (Calder et al. 2002), are an important source of immunoregulatory cytokines in the peripheral circulation and in localised foci of inflammation. TNF- $\alpha$ and IL- 6 synthesis by PBMC increases following immune activation and therefore these cytokines are potential markers with which to assess the functional effects of a putative anti-inflammatory intervention.

Eicosapentaenoic acid $(20: 5 n-3 ;$ EPA) and docosahexaenoic acid (22: $6 n-3$; DHA) are $n-3$ polyunsaturated fatty acids (PUFA) normally found in low concentrations in plasma and membrane phospholipids (Calder, 2001). EPA and DHA may regulate cell function, are substrates for the eicosanoid synthetic pathway and are metabolised to lipid peroxides (Sevanian \& Hochstein, 1985; Grimm et al. 2002). Dietary supplementation with fish oil, a rich source of EPA and DHA, results in increased incorporation of EPA and DHA into plasma, erythrocyte and PBMC phospholipids (Endres et al. 1989; Meydani et al. 1991a; Gallai et al. 1995; Caughey et al. 1996; Yaqoob et al. 2000) and increased

\footnotetext{
Abbreviations: EPA, eicosapentaenoic acid; DHA, docosahexaenoic acid; IL, interleukin; PBMC, peripheral blood mononuclear cells; PUFA, polyunsaturated fatty acid; TNF, tumour necrosis factor.

* Corresponding author: T. Trebble, fax +442380 794945, email timothy.trebble@btopenworld.com
} 
production of lipid peroxides (Haglund et al. 1991; Harats et al. 1991; Meydani et al. 1991b; Allard et al. 1997). There are conflicting reports of the effects of such alterations in phospholipid composition on PBMC function (Calder, 1997, 2001). Endres et al. (1989) and Meydani et al. (1991a) demonstrated inhibition of production of TNF- $\alpha$ and IL-1 but other investigators have failed to confirm this (Blonk et al. 1990; Cannon et al. 1995; Yaqoob et al. 2000; Thies et al. 2001a). There are a number of possible explanations for the inconsistencies in these reported results. First, the dose-response relationship between $n$-3 PUFA intake, EPA and DHA concentration in plasma and cell membrane phospholipids and cytokine production by PBMC has been poorly characterised, such that $n-3$ PUFA dietary supplementation studies have differed considerably in the quantities administered, ranging from $<1$ to $>4 \mathrm{~g} / \mathrm{d}$. Second, it is recognised that increased lipid peroxide production may have a pro-inflammatory effect on the immune system (Sevanian \& Hochstein, 1985), reduce in vivo concentrations of biological antioxidants such as vitamin E (Meydani et al. 1991b) and confound the anti-inflammatory effects of increased n-3 PUFA intake. The effect of antioxidant (vitamin E) co-supplementation on lipid peroxide production following fishoil consumption has been investigated in a number of studies (Haglund et al. 1991; Harats et al. 1991; Allard et al. 1997), but there are few results concerning the response of $n-3$ PUFA incorporation into phospholipid or PBMC function. As a consequence, studies have also varied in their inclusion of antioxidants in fish oil-supplementation studies.

The aims of the present study were to investigate the response of plasma and cell membrane phospholipid composition, PBMC function and lipid peroxidation to three levels of $n-3$ PUFA intake, chosen to represent the estimated mean habitual dietary intake in the UK population $(0.3 \mathrm{~g} / \mathrm{d})$, one recommended level of dietary intake $(1.0 \mathrm{~g} / \mathrm{d})$ and the intake provided by an oily-fish-rich meal $(2.0 \mathrm{~g} / \mathrm{d})$ (British Nutrition Foundation, 1999), with or without co-supplementation with the antioxidants ascorbic acid, $\alpha$-tocopheryl succinate, Se and Mn.

\section{Subjects and methods}

\section{Subjects}

The present study was approved by the Southampton and South West Hampshire Joint Research Ethics Committee. Healthy male subjects ( $n$ 16) were recruited from the staff of Southampton General Hospital and the University of Southampton. Subjects completed a brief lifestyle and medical history questionnaire and gave informed consent. A food-frequency questionnaire (validated in sixtyone adults; Shaheen et al. 2001) was completed to give an estimate of nutrient intake. Subjects were excluded if they were receiving any regular prescribed medication, had a history of hyperlipidaemia, hypertension or diabetes mellitus, or had a recent history of $n-3$ PUFA or antioxidant vitamin supplement use.

\section{Study design}

Subjects were allocated randomly, in a double-blind manner, to receive a daily antioxidant preparation $(200 \mu \mathrm{g} \mathrm{Se}, 3 \mathrm{mg}$ $\mathrm{Mn}, 30 \mathrm{mg} \mathrm{D}-\alpha$-tocopheryl succinate, $450 \mu \mathrm{g} \beta$-carotene and retinol (retinol equivalent) and $90 \mathrm{mg}$ ascorbic acid) or a placebo (maltose and lactose) that was identical in appearance (Wassen International, Leatherhead, Surrey, UK) for a period of 12 weeks. The antioxidant preparation was selected to represent a mix of vitamins and trace elements, each with recognised antioxidant properties. The preparation is commercially available. Simultaneously, all subjects received a 12-week course of fish oil containing EPA and DHA in the form of triacylglycerol (EPA-DHA (2:1, wt/wt; R. P. Scherer Ltd, Swindon, Wilts., UK)) and administered in $1 \mathrm{~g}$ capsules. The fish-oil doses administered to subjects were identical irrespective of whether they were receiving antioxidant co-supplementation or not, and consisted of $1 \mathrm{~g}$ fish $\mathrm{oil} / \mathrm{d}$ for the first 4 -week period, $3 \mathrm{~g} / \mathrm{d}$ for the second 4-week period and $6 \mathrm{~g} / \mathrm{d}$ for the third 4-week period, equivalent to a total EPA + DHA intake of $0.3,1.0$ and $2.0 \mathrm{~g} / \mathrm{d}$ respectively (Table 1). Each fish-oil capsule also contained $1 \mathrm{mg}$ vitamin E to stabilise the PUFA content. This quantity of vitamin $\mathrm{E}$ was considered insignificant and therefore unlikely to affect the results of the fish-oil only group. Subjects were asked to maintain their habitual diet throughout the intervention period, and to take the supplements every day prior to breakfast. Fasting peripheral venous blood samples were taken from the ante-cubital fossa at baseline (week 0), and after each 4-week period (weeks 4, 8 and 12). Subjects were reviewed every 4 weeks for side effects and issuing of fresh supplements. Assessment of compliance was by selfreporting.

\section{Cell and plasma preparation}

Blood samples were collected into heparin. Blood $(30 \mathrm{ml})$ was layered over $20 \mathrm{ml}$ Histopaque (density $1.077 \mathrm{~g} / \mathrm{ml}$;

Table 1. Intake of fish-oil capsules ( $n$ per d) and of individual polyunsaturated fatty acids $(\mathrm{mg} / \mathrm{d})$ from the fish oil during the intervention periods

\begin{tabular}{lcrrrrrrrr}
\hline & & \multicolumn{1}{c}{ Fatty acid intake $(\mathrm{mg} / \mathrm{d})$} \\
\cline { 3 - 10 } Week & Fish-oil capsules $\left(n\right.$ per d) ${ }^{*}$ & LN & LNA & AA & EPA & DPA & DHA & Total $n$-3 PUFA & Total $n$-6 PUFA \\
\hline $0-4$ & 1 & 12 & 9 & 11 & 208 & 24 & 101 & 342 & 26 \\
$4-8$ & 3 & 36 & 26 & 33 & 624 & 72 & 303 & 1025 & 78 \\
$8-12$ & 6 & 72 & 52 & 66 & 1248 & 144 & 606 & 2050 & 156 \\
\hline
\end{tabular}

LN, linoleic acid; LNA, $\alpha$-linolenic acid; AA, arachidonic acid; EPA, eicosapentaenoic acid; DPA, docosapentaenoic acid; DHA, docosahexaenoic acid; PUFA, polyunsaturated fatty acid.

${ }^{*}$ R. P. Scherer Ltd, Swindon Wilts., UK. 
Sigma-Aldrich Ltd, Poole, Dorset, UK) and centrifuged $\left(720 \mathrm{~g}\right.$ ) for $15 \mathrm{~min}$ at $20^{\circ} \mathrm{C}$. The plasma layer was removed and stored at $-70^{\circ} \mathrm{C}$. The PBMC layer was collected from the interface, washed and resuspended in medium (Roswell Park Memorial Institute medium containing 1.875 mM-glutamine and antibiotics). A second cycle was performed to reduce erythrocyte contamination and the cells were resuspended in $1 \mathrm{ml}$ medium, counted and removed for culture. For preparation of erythrocytes, $10 \mathrm{ml}$ blood was centrifuged $(720 \mathrm{~g})$ for $15 \mathrm{~min}$ at $4^{\circ} \mathrm{C}$. The plasma layer was removed and the cells washed with $0 \cdot 155 \mathrm{M}-\mathrm{NaCl}$.

\section{Fatty acid composition analysis}

Plasma (1 ml) and erythrocyte $(2 \mathrm{ml})$ samples were analysed. The internal standards dipentadecanoyl phosphatidylcholine (all samples) and diheptadecanoyl phosphatidylethanolamine (erythrocyte samples only) were added prior to analysis. Total plasma and cell lipids were isolated by extraction with chloroform-methanol $(2: 1, \mathrm{v} / \mathrm{v})($ Folch et al. 1957) containing $50 \mathrm{mg}$ butylated hydroxytoluene/l and individual lipid classes were purified by solid-phase extraction on aminopropylsilica cartridges (Varian, Walton-on-Thames, Surrey, UK) (Burdge et al. 2000). Purified lipids were converted to methyl esters by incubation with methanol containing $\mathrm{H}_{2} \mathrm{SO}_{4}(20 \mathrm{ml} / \mathrm{l})$ at $50^{\circ} \mathrm{C}$ for $18 \mathrm{~h}$ (Burdge et al. 2000). Fatty acid methyl esters were separated, re-dissolved in hexane and analysed by capillary GC using a Hewlett Packard 5890 GC (Hewlett Packard, Stockport, Ches., UK) equipped with an HP7686 GC autosampler with a BPX-70 fused silica capillary column $(50 \mathrm{~m} \times 0.32 \mathrm{~mm} \times 0.25 \mu \mathrm{m})$ with flame ionisation detection. Peaks were identified by retention times relative to standards. Plasma phosphatidylcholine, erythrocyte phosphatidylcholine and phosphatidylethanolamine fatty acids are reported as fractional concentrations $(\mathrm{g} / 100 \mathrm{~g}$ total fatty acids). For determination of phospholipid fatty acid composition $\mathrm{CV}$ was $<5 \%$.

\section{Cytokine analysis}

Purified PBMC at a concentration of $1 \times 10^{6}$ cells $/ \mathrm{ml}$ were incubated in $2 \mathrm{ml}$ medium containing autologous plasma $(50 \mathrm{ml} / \mathrm{l})$, with and without the monocyte-macrophage stimulant bacterial lipopolysaccharide at a concentration of $15 \mu \mathrm{g} / \mathrm{ml}$, for $24 \mathrm{~h}$. After this, culture plates were centrifuged $(180 \mathrm{~g})$ for $10 \mathrm{~min}$ at $20^{\circ} \mathrm{C}$ and the supernatant fraction removed and frozen at $-30^{\circ} \mathrm{C}$. Supernatant fractions were assayed in batches (all subject time points in a single batch) for cytokine concentrations (TNF- $\alpha$ and IL-6) using EASIA ${ }^{\mathrm{TM}}$ ELISA kits (Biosource Europe S.A., Nivelles, Belgium) according to the manufacturer's instructions. For both cytokine assays CV were $<10 \%$, and the limits of detection were $3 \mathrm{ng} / \mathrm{l}$ for TNF- $\alpha$ and $2 \mathrm{ng} / \mathrm{l}$ for IL-6.

\section{Malonaldehyde analysis}

Plasma malonaldehyde concentrations were determined by a colorimetric assay using a Bioxytech ${ }^{\circledR}$ LPO- $586^{\text {TM }}$ kit (Oxis International Inc., Portland, OR, USA), according to the manufacturer's instructions. $\mathrm{CV}$ was $<5 \%$.

\section{Statistical analysis}

Excel and Prism were used for data handling and graph generation, and SPSS (version 10; SPSS Inc., Chicago, IL, USA) and Stata (version 7; Stata Corp., College Station, TX, USA) were used for statistical analysis. Values are expressed as means with their standard errors for each population arm containing eight subjects, and as a pooled group of sixteen. All data were normally distributed, as determined by the Kolmogorov-Smirnov test. Statistical significance for the effects of antioxidant co-supplementation of $2.0 \mathrm{~g} / \mathrm{d}$ supplementary $n$-3 PUFA was determined using a univariate general linear model, with baseline values as a covariant. Groups and pooled data for the dose-response effects of $n-3$ PUFA were analysed using a Student's $t$ test for paired samples between baseline and following $2 \cdot 0 \mathrm{~g}$ supplementary $n-3$ PUFA/d. A post hoc analysis utilised identical statistical methods for intermediate intakes of $n$-3 PUFA. Phospholipid fatty acid composition changes and functional responses of PBMC were correlated using the general estimating equation, to allow for the repeated use of the population through the different intervention periods. Food-frequency questionnaires were compared in the two groups using a Student's $t$ test for independent samples. In all cases a value for $P<0.05$ was taken to indicate statistical significance.

\section{Results}

Subjects

All subjects completed the trial with compliance to the intervention regimen $>80 \%$. There were no major side effects of treatment, three subjects (two in antioxidant group, one in placebo group) reported mild hay fever (one as a first presentation). There were no significant differences between the fish-oil plus antioxidant and fishoil only groups with regard to habitual intake of $n-3$ or $n$-6 PUFA or antioxidants (Table 2), baseline $n-3$ PUFA concentrations in plasma or cell membrane, or baseline TNF- $\alpha$ or IL-6 production by PBMC.

\section{Fatty acid composition of plasma and cell phospholipids}

EPA and DHA incorporation into plasma phosphatidylcholine and erythrocyte phosphatidylethanolamine, and EPA into erythrocyte phosphatidylcholine, demonstrated a positive dose-dependent response to increasing $n$-3 PUFA intake, tending towards a plateau effect at higher levels (Table 3). In erythrocyte phosphatidylcholine, DHA incorporation decreased in response to intermediate supplementary intakes of $n$-3 PUFA, but increased non-significantly at the highest intakes. Docosapentaenoic acid (22:5n-3) incorporation significantly increased in plasma phosphatidylcholine in a dose-dependent manner, despite its relatively low concentration in the dietary supplements.

EPA incorporation into erythrocyte phosphatidylethanolamine was significantly higher in the fish-oil plus antioxidant group compared to the fish-oil only group at $1.0 \mathrm{~g} \mathrm{n-3}$ PUFA supplemental intake/d $(P=0.037)$ (allowing for baseline variability), although not at the $0 \cdot 3(P=0 \cdot 052)$ 
Table 2. Age and habitual dietary intakes of polyunsaturated fatty acids and antioxidants in the fish-oil plus antioxidant and fish-oil only groups*

(Mean values with their standard errors for eight subjects)

\begin{tabular}{|c|c|c|c|c|}
\hline \multirow[b]{2}{*}{ Intervention group... } & \multicolumn{2}{|c|}{ Fish-oil only } & \multicolumn{2}{|c|}{$\begin{array}{l}\text { Fish-oil plus } \\
\text { antioxidant }\end{array}$} \\
\hline & Mean & SEM & Mean & SEM \\
\hline Mean age (years) & $30 \cdot 6$ & 4.5 & $30 \cdot 3$ & $6 \cdot 1$ \\
\hline \multicolumn{5}{|l|}{ Dietary intake } \\
\hline Total $n-6$ PUFA (g/d) & $13 \cdot 5$ & $1 \cdot 4$ & $13 \cdot 4$ & $2 \cdot 5$ \\
\hline Total $n-3$ PUFA (g/d) & 1.9 & 0.06 & 1.8 & 0.3 \\
\hline Retinol $(\mu \mathrm{g} / \mathrm{d})$ & 434.6 & 99.8 & $449 \cdot 7$ & $88 \cdot 2$ \\
\hline Carotene $(\mu \mathrm{g} / \mathrm{d})$ & 3542 & 415 & 2819 & 490 \\
\hline Vitamin C (mg/d) & $181 \cdot 2$ & $31 \cdot 8$ & $167 \cdot 0$ & 33.9 \\
\hline Vitamin $E(\mathrm{mg} / \mathrm{d})$ & $11 \cdot 0$ & $1 \cdot 3$ & $11 \cdot 1$ & $1 \cdot 7$ \\
\hline $\operatorname{Se}(\mu \mathrm{g} / \mathrm{d})$ & $77 \cdot 6$ & $1 \cdot 7$ & $66 \cdot 3$ & $6 \cdot 8$ \\
\hline $\mathrm{Mn}(\mathrm{mg} / \mathrm{d})$ & $4 \cdot 8$ & 0.7 & 4.4 & 0.5 \\
\hline
\end{tabular}

PUFA, polyunsaturated fatty acid.

* Determined by a food-frequency questionnaire.

or $2.0 \mathrm{~g} / \mathrm{d}(P=0 \cdot 08)$ intakes. Similar trends were seen in other phospholipid pools.

\section{Cytokine production by cultured peripheral blood mononuclear cells}

$n$-3 PUFA supplementation of the diet decreased production of both TNF- $\alpha$ and IL- 6 by unstimulated and stimulated PBMC in both fish-oil only and fish-oil plus antioxidant groups (Figs 1 and 2). Antioxidant co-supplementation was associated with a trend towards greater proportionate reductions in TNF- $\alpha$ and IL- 6 synthesis by
PBMC at a supplementary $n-3$ PUFA intake of $0.3 \mathrm{~g} / \mathrm{d}$ compared with baseline. However, there were no significant differences in cytokine production by PBMC between the fish-oil plus antioxidant and fish-oil only groups at any supplementary $n-3$ PUFA intake. The two groups were therefore combined ( $n$ 16) for analysis of the doseresponse effects of $n$-3 PUFA intake on TNF- $\alpha$ and IL- 6 production (Table 4). In this unified group, the relationship between TNF- $\alpha$ and IL- 6 production by unstimulated and stimulated PBMC and increasing supplemental $n-3$ PUFA intake appeared to be characterised by a 'U-shaped' dose-response curve with maximum inhibitory effects noted at $1.0 \mathrm{~g} / \mathrm{d}$ compared with both 0.3 and $2.0 \mathrm{~g} / \mathrm{d}$. TNF- $\alpha$ production by unstimulated PBMC and IL- 6 production by stimulated PBMC were significantly lower at an $n-3$ PUFA intake of 1.0 compared with the $0.3 \mathrm{~g} / \mathrm{d}$ intake, but there was no significant difference found between 0.3 and $2.0 \mathrm{~g} / \mathrm{d}$ intakes. EPA concentrations in plasma and erythrocyte phosphatidylcholine and erythrocyte phosphatidylethanolamine were significantly negatively correlated with cytokine production by PBMC (Table 5).

\section{Plasma malonaldehyde concentration}

Plasma malonaldehyde concentration at baseline was 2.56 (SEM $0 \cdot 2) \mu \mathrm{mol} / \mathrm{l}(n$ 16). This was not significantly affected by consumption of $n$-3 PUFA alone or in combination with antioxidants, although there was a trend for increased production of malonaldehyde after a supplementary intake of $2.0 \mathrm{~g} n-3$ PUFA/d (2.97 (SEM 0.22) $\mu \mathrm{mol} / \mathrm{l}$, $P=0 \cdot 057)$.

Table 3. Fatty acid composition of plasma phosphatidylcholine (PC) and erythrocyte phosphatidylethanolamine (PE) and PC in response to increased $n-3$ polyunsaturated fatty acid (PUFA) intake as fish oil with and without antioxidant co-supplements§

(Mean values with their standard errors)

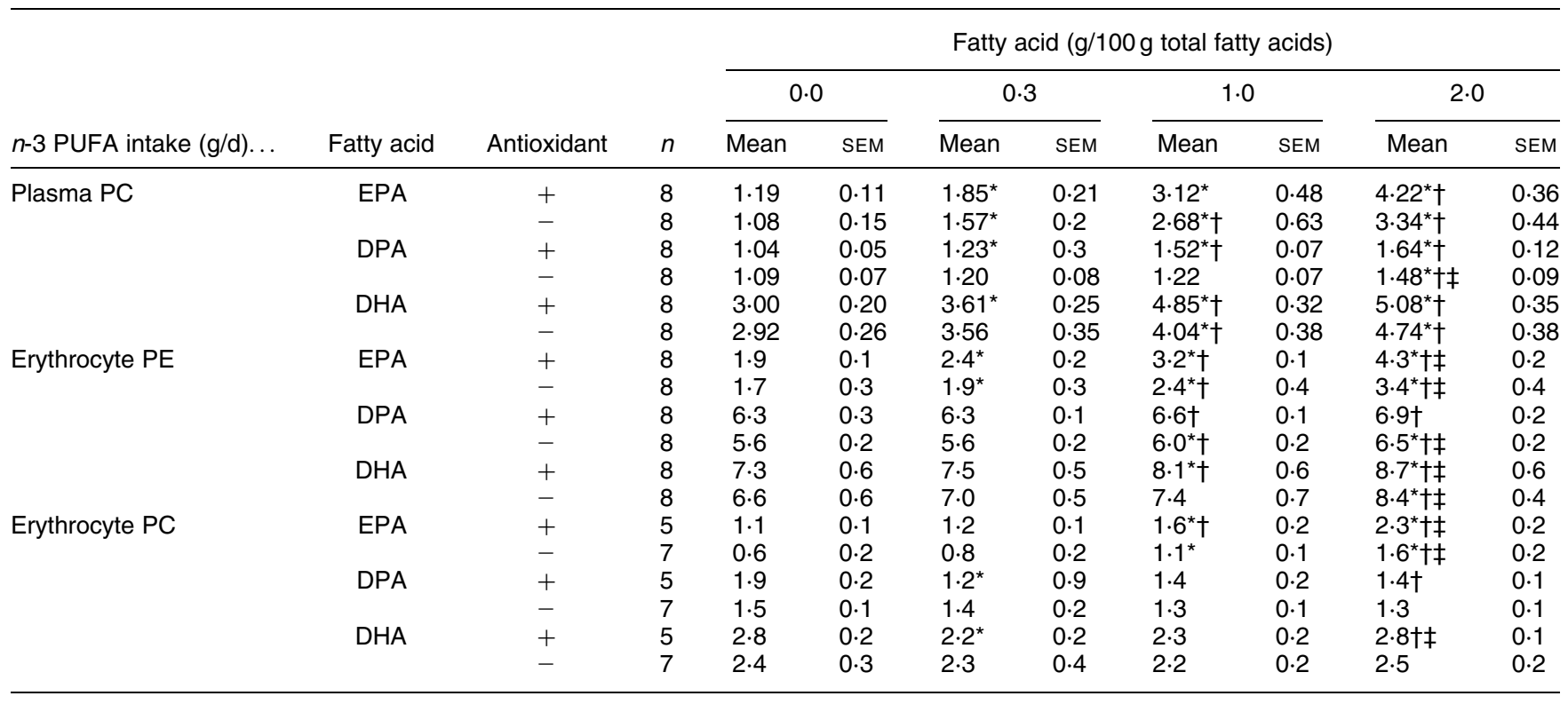

EPA, eicosapentaenoic acid; DPA, docosapentaenoic acid; DHA, docosahexaenoic acid.

Mean values were significantly different from those at baseline: ${ }^{*} P<0.05$.

Mean values were significantly different from those of the $0.3 \mathrm{~g}$ supplementary $n$-3 PUFA group intake: $\dagger P<0.05$.

Mean values were significantly different from those of the $1.0 \mathrm{~g}$ supplementary $n$-3 PUFA group intake: $\ddagger P<0.05$.

$\S$ For details of diets, supplements, subjects and procedures, see Tables 1 and 2 and p. 406. 
(a)

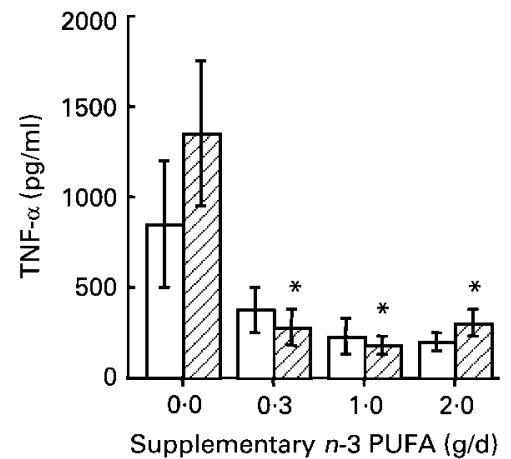

(b)

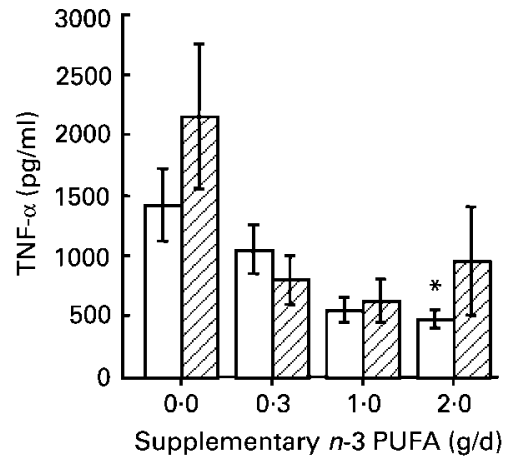

Fig. 1. Tumour necrosis factor (TNF)- $\alpha$ concentrations in culture supernatant fractions of (a) unstimulated and (b) lipopolysaccharide-stimulated peripheral blood mononuclear cells at baseline and in response to dietary fish-oil supplements providing $0.3,1.0$ and $2.0 \mathrm{~g} n-3$ polyunsaturated fatty acid (PUFA)/d. $\square$, Fish oil plus antioxidant; $\llbracket$, fish oil only. For details of diets, supplements, subjects and procedures, see Tables 1 and 2 and p. 406. Values are means with their standard errors shown by vertical bars $(n 8)$. Mean values were significantly different from those at baseline $(0.0 \mathrm{~g} / \mathrm{d}):{ }^{*} P<0.05$.

\section{Discussion}

Dietary supplementation with fish oil resulted in marked increases in the incorporation of EPA and DHA into plasma phosphatidylcholine and erythrocyte phosphatidylethanolamine in healthy male subjects. There was a positive dose-dependent relationship between EPA and DHA intake and their concentrations within the phospholipid pools, which tended towards a plateau at higher intakes. This finding is consistent with the results of previous studies (Blonk et al. 1990; Molvig et al. 1991; Healy et al. 2000). In erythrocyte phosphatidylcholine, EPA incorporation followed a similar pattern of response to phosphatidylethanolamine changes, but DHA incorporation decreased at intermediate $n$-3 PUFA supplementary intakes and was not significantly different from baseline at the $2.0 \mathrm{~g} / \mathrm{d}$ intake.

Dietary fish-oil supplementation resulted in significant reductions in TNF- $\alpha$ and IL- 6 production by PBMC, even at the lowest supplementary intake equivalent to $0.3 \mathrm{~g} \mathrm{n}-3$ PUFA/d. The relationship between $n-3$ PUFA and cytokine production by the PBMC appeared to conform to a negative but 'U-shaped' dose-response relationship, with maximum inhibition demonstrated at a supplementary intake of

(a)

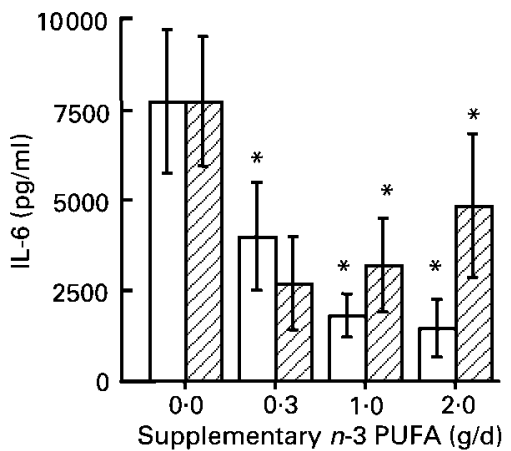

$1.0 \mathrm{~g} / \mathrm{d}$. TNF- $\alpha$ and IL-6 production by PBMC correlated negatively with EPA concentrations in plasma and erythrocyte phospholipid.

Plasma concentrations of the lipid peroxide malonaldehyde demonstrated a trend towards a dose-dependent increase with increasing supplementary $n-3$ PUFA intake, consistent with its proposed mechanism of production. Antioxidant co-supplementation augmented the increase in EPA incorporation into erythrocyte phospholipid at an $n-3$ PUFA intake of $1.0 \mathrm{~g} / \mathrm{d}$, but without any alteration in the production of lipid peroxides. These findings contrast with the inhibitory effects of vitamin E dietary supplementation on lipid peroxide generation noted in previous studies using similar doses (Haglund et al. 1991; Meydani et al. 1991b; Palozzo et al. 1996), but comparisons are complicated both by the different quantities of fish oil used in each of these studies and the additional antioxidants co-administered in the current study. Antioxidant co-supplementation amplified the decreases in cytokine production by PBMC at an $n-3$ PUFA supplementary intake of $0 \cdot 3 \mathrm{~g} / \mathrm{d}$, but did not result in any absolute differences in TNF- $\alpha$ or IL-6 production between the two groups at any intake.

(b)

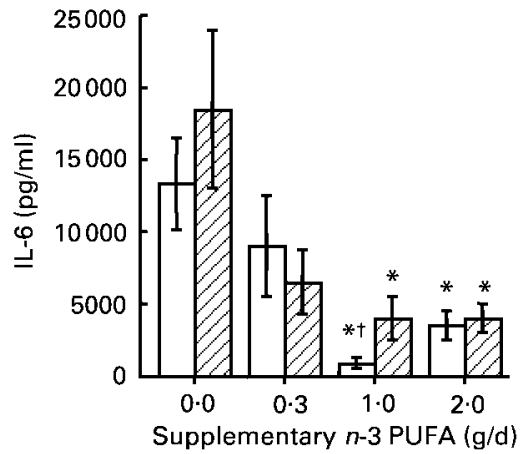

Fig. 2. Interleukin (IL) 6 concentrations in culture supernatant fractions of (a) unstimulated and (b) lipopolysaccharide-stimulated peripheral blood mononuclear cells at baseline and in response to dietary fish-oil supplements providing $0.3,1.0$ and $2.0 \mathrm{~g} n$-3 polyunsaturated fatty acid (PUFA)/d. $\square$, Fish oil plus antioxidant; $\llbracket$, fish oil only. For details of diets, supplements, subjects and procedures, see Tables 1 and 2 and p. 406. Values are means with their standard errors shown by vertical bars $(n 8)$. Mean values were significantly different from those at baseline $(0.0 \mathrm{~g} / \mathrm{d}):{ }^{*} P<0.05$. Mean value was significantly different from that of the $0.3 \mathrm{~g}$ supplementary $n-3 \mathrm{PUFA} / \mathrm{d}$ group: $\dagger P<0.05$. 
Table 4. Tumour necrosis factor (TNF)- $\alpha$ and interleukin (IL) 6 concentrations in culture supernatant fractions of unstimulated and lipopolysaccharide-stimulated peripheral blood mononuclear cells in response to supplementary intakes of $n-3$ polyunsaturated fatty acids (PUFA), as fish oil, in healthy subjects $\neq$

(Mean values with their standard errors for sixteen subjects)

\begin{tabular}{|c|c|c|c|c|c|c|c|c|c|}
\hline \multirow[b]{3}{*}{$n-3$ PUFA intake $(\mathrm{g} / \mathrm{d}) \ldots$} & & \multicolumn{8}{|c|}{ Cytokine (pg/ml) } \\
\hline & & \multicolumn{2}{|c|}{0.0} & \multicolumn{2}{|c|}{0.3} & \multicolumn{2}{|c|}{$1 \cdot 0$} & \multicolumn{2}{|c|}{$2 \cdot 0$} \\
\hline & & Mean & SEM & Mean & SEM & Mean & SEM & Mean & SEM \\
\hline \multirow[t]{2}{*}{ TNF- $\alpha$} & Unstimulated & 1134 & 221 & $320^{\star *}$ & 61 & $154^{\star \star} \dagger$ & 29 & $187^{\star \star}$ & 29 \\
\hline & Stimulated & 1787 & 368 & $929^{*}$ & 126 & $607^{*}$ & 137 & $725^{\star}$ & 216 \\
\hline \multirow[t]{2}{*}{ IL-6 } & Unstimulated & 7888 & 1209 & $3735^{\star}$ & 713 & $2734^{\star \star}$ & 660 & $3251^{\star *}$ & 1131 \\
\hline & Stimulated & 16220 & 2911 & $7916^{*}$ & 1904 & $2630^{* \star} \dagger$ & 864 & $3348^{\star \star} \dagger$ & 799 \\
\hline
\end{tabular}

Mean values were significantly different from those at baseline: ${ }^{\star} P<0.05 ;{ }^{\star \star} P<0.01$.

Mean values were significantly different from those of the $0.3 \mathrm{~g}$ supplementary $n$-3 PUFA group: $\dagger P<0.05$.

$\ddagger$ For details of diets, supplements, subjects and procedures, see Tables 1 and 2 and p. 406

Estimated habitual dietary intakes in the fish-oil plus antioxidant and fish-oil only groups were equivalent to 1.8 and $1.9 \mathrm{~g} \mathrm{n-3}$ PUFA/d and 13.4 and $13.5 \mathrm{~g} \mathrm{n-6}$ PUFA/d respectively, values that are in accordance with the estimated mean UK adult intakes (Committee on Medical Aspects of Food Policy, 1991). n-3 PUFA intake in the UK diet is primarily as $\alpha$-linolenic acid, with much smaller amounts of EPA and DHA being consumed. In the present study population, dietary supplementation with fish oil resulted in an increase in $n$-3 PUFA intake of $0.3,1.0$ and $2.0 \mathrm{~g} / \mathrm{d}$, but principally as EPA and DHA.

Several previous studies have described functional inhibition of PBMC and other immune cells at supplemental n-3 PUFA intakes > 4 g/d (Lee et al. 1985; Endres et al. 1989; Molvig et al. 1991), but the results of other studies have been inconsistent (for review, see Calder, 2001). As well as confirming the postulated anti-inflammatory effects of $n$-3 PUFA dietary supplementation, we have described for the first time a dose-response relationship between n-3 PUFA dietary intake, EPA concentration within phospholipid pools and TNF- $\alpha$ and IL- 6 production by PBMC. We have also demonstrated for the first time inhibitory effects on cytokine release by PBMC at supplementary $n-3$ PUFA intakes $<1 \mathrm{~g} / \mathrm{d}$. Some studies have demonstrated that increasing $n-3$ PUFA intake results in a dose-dependent increase in EPA concentrations in plasma and leucocyte phospholipid (Blonk et al. 1990; Palozza et al. 1996; Healy et al. 2000), but attempts to characterise the relationship to immune cell function have failed due to absent or inconsistent effects on the responses investigated (Blonk et al. 1990; Molvig et al. 1991; Healy et al. 2000).

The described dose-response relationships between $n-3$ PUFA intake, phospholipid composition and cytokine production by PBMC may explain some of the inconsistent results of previous dietary fish-oil supplement studies. They may also offer a plausible explanation for the observations by Endres et al. (1989) that TNF- $\alpha$ production by PBMC from healthy subjects did not alter following 6 weeks of dietary supplementation with fish oil (equivalent to $4 \mathrm{~g} n-3$ PUFA/d), but decreased 10 weeks after subjects returned to their normal habitual intake. Importantly, at the 10-week time point, EPA concentrations within phospholipid remained $30 \%$ above baseline values with a significantly decreased arachidonic acid:EPA ratio approximately mid-way between the ratio at the end of the supplementation period and that at baseline (Endres et al. 1989). If the dose-response relationship is 'U-shaped', as suggested in the current study, an intermediate concentration of EPA within plasma and cell membrane phospholipid, resulting from a supplemental $n-3$ PUFA intake of $<2.0 \mathrm{~g} / \mathrm{d}$, may be associated with a greater inhibitory effect on $\mathrm{TNF}-\alpha$ release than higher EPA concentrations resulting from $n-3$ PUFA supplementary intakes of $>2.0 \mathrm{~g} / \mathrm{d}$. Extrapolating this finding to subjects in the study by Endres et al. (1989), a greater inhibition of TNF$\alpha$ production was seen at intermediate EPA concentrations

Table 5. Regression co-efficients $(\mathrm{RC})$ and correlation co-efficients $(r)$ for the relationships between eicosapentaenoic acid in plasma phosphatidylcholine (PC), erythrocyte phosphatidylethanolamine (PE) or erythrocyte PC and production of tumour necrosis factor (TNF)- $\alpha$ and interleukin (IL) 6 by unstimulated and lipopolysaccharide (LPS)-stimulated peripheral blood mononuclear cells from healthy subjects ( $n$ 16) over a range of supplemental $n-3$ PUFA intakes $(0.0$ to $2.0 \mathrm{~g} / \mathrm{d}) \dagger$

\begin{tabular}{|c|c|c|c|c|c|c|c|}
\hline \multirow[b]{2}{*}{ Cytokine } & \multirow[b]{2}{*}{ LPS } & \multicolumn{2}{|c|}{ Plasma PC } & \multicolumn{2}{|c|}{ Erythrocyte PE } & \multicolumn{2}{|c|}{ Erythrocyte PC } \\
\hline & & $\mathrm{RC}$ & $r$ & $\mathrm{RC}$ & $r$ & $\mathrm{RC}$ & $r$ \\
\hline TNF- $\alpha$ & $\begin{array}{l}- \\
+\end{array}$ & $\begin{array}{l}-0.67 \\
-0.43\end{array}$ & $\begin{array}{l}-0.37^{\star \star} \\
-0.22^{\star}\end{array}$ & $\begin{array}{l}-0.65 \\
-0.07\end{array}$ & $\begin{array}{l}-0.24^{*} \\
-0.22^{\star}\end{array}$ & $\begin{array}{l}-0.94 \\
-0.83\end{array}$ & $\begin{array}{l}-0.39^{\star *} \\
-0.42^{\star *}\end{array}$ \\
\hline IL-6 & $\begin{array}{l}- \\
+\end{array}$ & $\begin{array}{l}-17.5 \\
-29.9\end{array}$ & $\begin{array}{l}-0.30^{\star \star} \\
-0.36^{\star *}\end{array}$ & $\begin{array}{l}-21 \cdot 51 \\
-40 \cdot 1\end{array}$ & $\begin{array}{l}-0.22^{*} \\
-0.33^{\star *}\end{array}$ & $\begin{array}{l}-29 \cdot 4 \\
-40 \cdot 8\end{array}$ & $\begin{array}{l}-0.45^{\star \star} \\
-0.36^{\star \star}\end{array}$ \\
\hline
\end{tabular}

${ }^{\star} P<0.05 ;{ }^{* \star} P<0.01$.

†For details of diets, supplements, subjects and procedures, see Tables 1 and 2 and p. 406. 
in PBMC lipid at the 10-week time point than at higher EPA concentrations associated with 4.0 g n-3 PUFA dietary supplementation/d. Furthermore, Endres et al. (1989) and others (Molvig et al. 1991) noted that cessation of this inhibitory effect on cytokine synthesis by PBMC occurred when EPA concentrations within phospholipid returned to baseline values. This suggests that the inhibitory effects of dietary $n$-3 PUFA on PBMC function reflect phospholipid concentration of EPA and not the time course of supplementation or previous intake of $n-3$ PUFA; this is also supported by the correlations noted earlier between cell membrane phospholipid content and TNF- $\alpha$ production.

In the current study, the use of an open trial design to investigate changes in cytokine production by PBMC involved the comparison, in each subject, of values at each time point with the value at baseline. This reduced the confounding effects of wide inter-individual variations in TNF- $\alpha$ and IL-6 production (Jacob et al. 1990; Yaqoob et al. 1999), as commonly complicate parallel studies. Intra-individual variation in cytokine production by comparison is relatively small (Jacob et al. 1990; Yaqoob et al. 1999). We omitted 'wash-out' periods between interventions as the phospholipid concentrations of EPA increased at each time point, therefore negating the effect of previous levels of supplementation. Furthermore, maximal changes in the fatty acid composition of plasma and PBMC occur within 4 weeks of increasing intake (Gibney \& Hunter, 1993; Yaqoob et al. 2000; Thies et al. $2001 b$ ), which was therefore chosen as the intervention period for each level of dietary $n-3$ PUFA supplementation in our present study. The 'wash-out' period required to return $n$-3 PUFA concentrations to baseline values after each intervention may be as long as 20 weeks (Endres et al. 1989). If included in our present trial design, this would have increased the time course of the study to approximately 1 year, and introduced inaccuracy due to the effects of seasonally related variations in cytokine production (Maes et al. 1994).

The mechanism of action of dietary $n-3$ PUFA on the PBMC remains unexplained. It is postulated that increased $n$-3 PUFA concentrations in the cell membrane are associated with both stimulatory and inhibitory effects on cell function, ranging from modulation of intercellular eicosanoid release to alterations in gene transcription (De Pablo $\&$ De Cienfuegos, 2000). These factors could result in a 'U-shaped' dose-response curve if their maximum effect occurred at different intakes of $n$-3 PUFA.

There are important clinical implications of the results of the present study. First, the characterisation of a doseresponse relationship between supplemental $n$-3 PUFA intake and PBMC function, and recognition of the influence of antioxidant co-supplementation on EPA incorporation into phospholipid, is valuable pharmacological data required for the development of $n$-3 PUFA as a clinical intervention. Second, that anti-inflammatory efficacy is possible with a supplementary $n-3$ PUFA intake as low as $1.0 \mathrm{~g} / \mathrm{d}$ might reduce cost and side effects of treatment and therefore increase their attractiveness to physicians and patients.

In conclusion, supplemental $n$-3 PUFA intakes between 0.3 and $2.0 \mathrm{~g} / \mathrm{d}$ in healthy subjects result in defined but contrasting patterns of response with regard to phospholipid composition and PBMC cytokine production. Antioxidant co-supplementation appears to increase the incorporation of $n$-3 PUFA into phospholipid at supplemental intakes of $<2.0 \mathrm{~g} / \mathrm{d}$ and augments the inhibitory effect of supplementary $n-3$ PUFA intakes $<1.0 \mathrm{~g} / \mathrm{d}$ on the PBMC.

\section{Acknowledgements}

This work was supported by grants to T. T. from Hope (Wessex Medical Trust), Nutricia Clinical Care and the Southampton Rheumatology Trust. Fish-oil supplements were provided by R. P. Scherer Ltd and antioxidant supplements by Wassen plc.

\section{References}

Allard JP, Kurian R, Aghdassi E, Muggli R \& Royall D (1997) Lipid peroxidation during $n-3$ fatty acid and vitamin $\mathrm{E}$ supplementation in humans. Lipids 32, 535-541.

Blonk MC, Bilo HJG, Naua JJP, Popp-Snijders C, Mulder C \& Donker AJM (1990) Dose response effects of fish oil supplementation in healthy volunteers. Am J Clin Nutr 52, $120-127$.

British Nutrition Foundation (1999) Briefing Paper: n-3 Fatty Acids and Health. London: BNF.

Burdge GC, Wright P, Jones AE \& Wootton SA (2000) A method for separation of phosphatidylcholine, triacylglycerol, nonesterified fatty acids and cholesterol esters from plasma by solid-phase extraction. Br J Nutr 84, 781-787.

Calder PC (1997) n-3 Polyunsaturated fatty acid and cytokine production in health and disease. Ann Nutr Metabol 41, $203-234$.

Calder PC (2001) n-3 Polyunsaturated fatty acids, inflammation and immunity: pouring oil on troubled water or another fishy tale? Nutr Res 21, 309-341.

Calder PC, Yaqoob P, Thies F, Wallace FA \& Miles EA (2002) Fatty acids and lymphocyte functions. Br J Nutr 87, Suppl. 1, S31-S48.

Cannon JG, Fiatarone MA, Meydani M, et al. (1995) Aging and dietary modulation of elastase and interleukin-1 beta secretion. Am J Physiol 268, R208-R213.

Caughey GE, Mantzioris E, Gibson RA, Cleland LG \& James MJ (1996) The effect on human tumor necrosis factor alpha and interleukin 1 beta production of diets enriched in $n-3$ fatty acids from vegetable oil or fish oil. Am J Clin Nutr 63, $116-122$.

Committee on Medical Aspects of Food Policy (1991) Dietary Reference Values for Food Energy and Nutrients for the United Kingdom. Report on Health and Social Subjects. no. 41 London: H. M. Stationery Office.

De Pablo M \& De Cienfuegos GA (2000) Modulatory effects of dietary lipids on immune system functions. Immunol Cell Biol 78, $31-39$.

Elliott MJ, Feldmann M \& Maini RN (1995) TNF alpha blockade in rheumatoid arthritis: rationale, clinical outcomes and mechanisms of action. Int $J$ Immunopharmacol 17, $141-145$.

Endres S, Ghorbani R, Kelley VE, et al. (1989) The effect of dietary supplementation with $n-3$ polyunsaturated fatty acids on the synthesis of interleukin-1 and tumor necrosis factor by mononuclear cells. New Eng J Med 320, 265-271.

Folch J, Lees M \& Sloane-Stanley GH (1957) A simple method for the isolation and purification of total lipides from animal tissues. J Biol Chem 226, 497-509. 
Gallai V, Sarchielli P, Trequattrini A, et al. (1995) Cytokine secretion and eicosanoid production in the peripheral blood mononuclear cells of MS patients undergoing dietary supplementation with $n-3$ polyunsaturated fatty acids. J Neuroimmunol 56, $143-153$.

Gibney MJ \& Hunter B (1993) The effects of short- and long-term supplementation with fish oil on the incorporation of $n-3$ polyunsaturated fatty acids into cells of the immune system in healthy volunteers. Eur J Clin Nutr 47, 255-259.

Grimm H, Mayer K, Mayser P \& Eigenbrodt E (2002) The regulatory potential of $n-3$ fatty acids in immunological and inflammatory processes. Br J Nutr 87, Suppl. 1, S59-S67.

Haglund O, Luostarinen R, Wallin R, Wibell L \& Saldeen T (1991) The effects of fish oil on triglycerides, cholesterol, fibrinogen and malonaldehyde in humans supplemented with vitamin E. J Nutr 121, 165-169.

Harats D, Dabach Y, Hollander G, et al. (1991) Fish oil ingestion in smokers and nonsmokers enhances peroxidation of plasma lipoproteins. Atheroscherosis 90, 127-139.

Healy DA, Wallace FA, Miles EA, Calder PC \& Newsholme P (2000) Effect of low-to-moderate amounts of dietary fish oil on neutrophil lipid composition and function. Lipids 35, $763-768$.

Jacob CO, Fronek Z, Lewis GD, Koo M, Hansen JA \& McDevitt HO (1990) Heritable major histocompatibility complex class II-associated differences in production of tumor necrosis factor alpha: relevance to genetic predisposition to systemic lupus erythematosus. Proc Natl Acad Sci USA 87, $1233-1237$.

Lee TH, Hoover RL, Williams JD, et al. (1985) Effect of dietary enrichment with eicosapentaenoic and docosahexaenoic acids on in vitro neutrophil and monocyte leukotriene generation and neutrophil function. New Eng J Med 312, 1217-1224.

Maes M, Stevens W, Scharpe S, et al. (1994) Seasonal variation in peripheral blood leukocyte subsets and in serum interleukin6 , and soluble interleukins-2 and -6 receptor concentrations in normal volunteers. Experientia 50, 821-829.

Meydani SN, Endres S, Woods MM, et al. (1991a) Oral (n-3) fatty acid supplementation suppresses cytokine production and lymphocyte proliferation: comparison between young and older women. J Nutr 121, 547-555.

Meydani M, Natiello F, Goldin B, et al. (1991b) Effect of long-term fish oil supplementation on vitamin E status and lipid peroxidation in women. J Nutr 121, 484-491.

Molvig J, Pociot F, Worsaae H, et al. (1991) Dietary supplementation with omega-3-polyunsaturated fatty acids decreases mononuclear cell proliferation and interleukin-1 beta content but not monokine secretion in healthy and insulin-dependent diabetic individuals. Scand J Immunol 34, 399-410.

Palozzo P, Sgarlata E, Luberto C, et al. (1996) n-3 Fatty acids induce oxidative modifications in human erythrocytes depending on dose and duration of dietary supplementation. Am J Clin Nutr 64, 297-304.

Sevanian A \& Hochstein P (1985) Mechanisms and consequences of lipid peroxidation in biological systems. Annu Rev Nutr $\mathbf{5}$, 365-390.

Shaheen SO, Sterne JAC, Thompson RL, Songhurst CE, Margetts BM \& Burney PGJ (2001) Dietary antioxidants and asthma in adults. Population-based case-control study. Am J Respir Crit Care Med 164, 1823-1828.

Targan SR, Hanauer SB, van Deventer SJ, et al. (1997) A short-term study of chimeric monoclonal antibody cA2 to tumor necrosis factor alpha for Crohn's disease. Crohn's Disease cA2 Study Group. New Eng J Med 337, 1029-1035.

Thies F, Miles EA, Nebe-von-Caron G, Powell JR, et al. (2001a) Influence of dietary supplementation with long-chain $n-3$ or $n-6$ polyunsaturated fatty acids on blood inflammatory cell populations and functions and on plasma soluble adhesion molecules in healthy humans. Lipids 36, 1183-1193.

Thies F, Nebe-von-Caron G, Powell JR, Yaqoob P, Newsholme EA \& Calder PC (2001b) Dietary supplementation with gamma linolenic acid or fish oil decreases $\mathrm{T}$ lymphocyte proliferation in healthy older humans. $J$ Nutr 131, 1918-1927.

Yaqoob P, Newsholme EA \& Calder PC (1999) Comparison of cytokine production in cultures of whole human blood and purified mononuclear cells. Cytokine 11, 600-605.

Yaqoob P, Pala HS, Cortina-Borja M, Newsholme EA \& Calder PC (2000) Encapsulated fish oil enriched in alpha-tocopherol alters plasma phospholipid and mononuclear cell fatty acid compositions but not mononuclear cell functions. Eur $J$ Clin Invest 30, 260-274. 